\title{
A novel biosensor to monitor proline in pea root exudates and nodules under osmotic stress and recovery
}

\author{
María I. Rubia • Vinoy K. Ramachandran • \\ Cesar Arrese-Igor • Estíbaliz Larrainzar • \\ Philip S. Poole
}

Received: 7 October 2019 / Accepted: 19 May 2020 /Published online: 4June2020

(C) The Author(s) 2020

\begin{abstract}
Background and aims Plant and bacteria are able to synthesise proline, which acts as a compound to counteract the negative effects of osmotic stresses. Most methodologies rely on the extraction of compounds using destructive methods. This work describes a new proline biosensor that allows the monitoring of proline levels in a non-invasive manner in root exudates and nodules of legume plants.

Methods The proline biosensor was constructed by cloning the promoter region of pRL120553, a gene with high levels of induction in the presence of proline, in front of the lux cassette in Rhizobium leguminosarum bv. viciae.

Results Free-living assays show that the proline biosensor is sensitive and specific for proline. Proline was detected in both root exudates and nodules of pea plants. The
\end{abstract}

In memory of Prof. Tomás Ruiz-Argüeso, for whom we once were "offensively young", for his generosity transmitting both scientific knowledge and passion.

Euan K. James

Electronic supplementary material The online version of this article (https://doi.org/10.1007/s11104-020-04577-2) contains supplementary material, which is available to authorized users.

M. I. Rubia · C. Arrese-Igor · E. Larrainzar $(\bowtie)$

Institute for Multidisciplinary Research in Applied

Biology-IMAB, Universidad Pública de Navarra, Campus

Arrosadia, Pamplona 31006, Spain

e-mail: estibaliz.larrainzar@unavarra.es

V. K. Ramachandran · P. S. Poole $(\bowtie)$

Department of Plant Sciences, University of Oxford, Oxford OX1 3RB, UK

e-mail: philip.poole@plants.ox.ac.uk luminescence detected in bacteroids did not show variations during osmotic stress treatments, but significantly increased during recovery.

Conclusions This biosensor is a useful tool for the in vivo monitoring of proline levels in root exudates and bacteroids of symbiotic root nodules, and it contributes to our understanding of the metabolic exchange occurring in nodules under abiotic stress conditions.

Keywords Rhizosphere · Proline dehydrogenase · Water deficit · Salt stress $\cdot$ Rhizobium $\cdot$ Symbiosis
Abbreviations
ANA apparent nitrogenase activity
CFU colony-forming units
cps counts per second
dpi days post inoculation
P5C pyrroline-5-carboxylate
P5CDH delta-1-pyrroline-5-carboxylate dehydrogenase
ProDH proline dehydrogenase
RLU relative luminescence units
$\mathrm{OD}_{600}$ optical density at $600 \mathrm{~nm}$
UMA universal minimal agar
UMS universal minimal salts

\section{Introduction}

Drought and salinity stress are some of the environmental factors most affecting plant growth and crop yield worldwide. In order to counteract the negative effects of 
osmotic stresses, plant and bacteria are able to synthesise osmoprotectant compounds to maintain cell viability. The amino acid proline, being highly soluble in water and a scavenger of reactive oxygen species, has been thought to provide protection under salt and waterdeficit stresses (Aspinall and Paleg 1981; Hasegawa et al. 2000; Szabados and Savouré 2010; Verdoy et al. 2006). In plants, proline catabolism is mediated by two enzymes; proline dehydrogenase (ProDH) producing pyrroline-5-carboxylate (P5C) from proline, and delta1-pyrroline-5-carboxylate dehydrogenase (P5CDH), which converts P5C to glutamate (Szabados and Savouré 2010). In bacteria, however, both steps are catalysed by a single polypeptide encoded by the gene putA, whose expression is regulated by putR and is induced in response to proline (Jimenez-Zurdo et al. 1997; Keuntje et al. 1995; Kohl et al. 1988). Besides its role as an osmoprotectant, proline catabolism has been also suggested to serve as an energy, carbon and nitrogen source under environmental stress conditions (Lee et al. 2009; Tanner 2008; van Overbeek and van Elsas 1995; Vives-Peris et al. 2018). Additionally, proline exudation has been shown to have a chemotactic effect in alfalfa roots (Bais et al. 2006; Webb et al. 2014). Monitoring proline utilisation in both plant and bacterial systems is a first key step towards understanding the multiple roles of this molecule under osmotic stress situations.

The rhizosphere is the nutrient-rich zone of soil in close proximity with the plant root system where microbial communities depend on the release of root exudates (Turner et al. 2013). Plant root exudates are composed of a great variety of primary and secondary metabolites, including low-molecular weight compounds such as sugars, amino acids and organic acids, as well as high-molecular weight molecules such as mucilage and proteins (Bais et al. 2006; Oburger and Jones 2018). The different molecules present in root exudates can mediate both positive and negative interactions in the rhizosphere (Huang et al. 2014; Olanrewaju et al. 2019). Focusing on the former, the symbiosis established between plants of the Leguminosae family and a group of alpha-proteobacteria named rhizobium has been widely studied. During this interaction, rhizobia are able to infect root cells through a complex signal exchange process, which requires the transcriptional reprogramming of roots cells to develop an organ specialized in nitrogen fixation named the nodule. The symbiosis between pea (Pisum sativum) plants and Rhizobium leguminosarum bv. viciae bacteria is a wellestablished model system to understand this plantmicrobe interaction (Oldroyd et al. 2011; Udvardi and Poole 2013) and has been effectively used to analyse the effect of root exudates in bacterial gene expression (Ramachandran et al. 2011). This transcriptomic analysis led to the identification of a number of bacterial genes specifically induced in response to certain solutes. Cloning the promoter regions of such genes upstream of the lux operon, Pini et al. (2017) generated a suite of luminescencebased bacterial bioreporters for the specific detection of metabolites in the rhizosphere. These biosensors allow real time monitoring of the release of a number of compounds including sugars, polyols, organic acids and amino acids in a non-destructive semi-quantitative manner, avoiding possible artefacts associated with other methodologies (Oburger and Jones 2018; Rilling et al. 2019, and references therein). Plants are grown on plates and, upon inoculation of the specific biosensor, the presence and the abundance of a specific compound can be monitored over time using a photon counting CCD camera.

In the current work, we extend this elite set of biosensors by describing a new lux biosensor for the detection of the amino acid proline. The construct relies on the expression of the lux reporter driven by the promoter of the pRL120553, a gene located in the proximity to the gene putA, responsible for proline catabolism in gram-negative bacteria (Jiménez-Zurdo et al. 1995; Liu et al. 2017). We monitored the levels of luminescence of the biosensor in pea roots and during nodulation both under optimal growth conditions and upon the application of water-deficit or salt stress. Our results show that, in bacteroids, proline accumulation does not occur during the stress phase, but during recovery, once optimal plant growth conditions are re-established.

\section{Materials and methods}

Bacterial strains and growth conditions

The bacterial strains and plasmids used in this study are listed in Table 1. R. leguminosarum bv. viciae 
Table 1 Bacterial strains and plasmids used in this work. Tc, tetracycline

\begin{tabular}{|c|c|c|c|c|}
\hline $\begin{array}{l}\text { R. leguminosarum } \\
\text { bv. viciae } 3841 \\
\text { strain (plasmid) }\end{array}$ & Description of the strain & Description of the plasmid & Resistance & Source \\
\hline LMB542 (pIJ11268) & $\begin{array}{l}\text { Strain with no lux expression used } \\
\text { as a negative control. }\end{array}$ & $\begin{array}{l}\text { Plasmid derived from pJP2, containing the } l u x \\
\text { operon with no promoter }\end{array}$ & $\mathrm{Tc}$ & Frederix et al. 2014 \\
\hline D5250 (pIJ11282) & $\begin{array}{l}\text { Strain with constitutive lux expression } \\
\text { used as a positive control. }\end{array}$ & $\begin{array}{l}\text { pIJ11268 with the promoter region of } n p t I I \\
\text { cloned upstream of the luxCDABE operon }\end{array}$ & $\mathrm{Tc}$ & Frederix et al. 2014 \\
\hline OPS0650 (pOPS0238) & Proline biosensor strain & $\begin{array}{l}\text { pIJ11268 with the promoter region of the gene } \\
\text { pRL120553 and the divergent gene } \\
\text { pRL120552 (putR) cloned upstream } \\
\text { of the luxCDABE operon }\end{array}$ & $\mathrm{Tc}$ & This work \\
\hline
\end{tabular}

3841 strains were grown at $28{ }^{\circ} \mathrm{C}$ in tryptone yeast agar or broth (Beringer 1974) or universal minimal agar supplemented with $30 \mathrm{mM}$ pyruvate and $10 \mathrm{mM}$ ammonium chloride as the carbon and nitrogen sources, respectively. Universal minimal salt medium (UMS) is based on the acid minimal salts (AMS; Poole et al. 1994) medium with the addition of EDTA- $\mathrm{Na}_{2}(1 \mu \mathrm{M}), \mathrm{CoCl}_{2} \mathrm{O} \cdot 6 \mathrm{H}_{2} \mathrm{O}(4.2 \mu \mathrm{M})$, $\mathrm{FeSO}_{4} \mathrm{O} \cdot 7 \mathrm{H}_{2} \mathrm{O}(0.04 \mathrm{mM})$, and $\mathrm{CaCl}_{2} \mathrm{O} \cdot 2 \mathrm{H}_{2} \mathrm{O}(0.51$ $\mathrm{mM}) .16 \mathrm{~g} \mathrm{~L}^{-1}$ agar was used for solid medium. Antibiotics were added to the cultures at the following concentrations $\left(\mu \mathrm{g} \mathrm{mL}^{-1}\right)$ : streptomycin, 500; tetracycline, 2.

For the construction of the proline biosensor, the promoter region of pRL120553 (605 bp, including the complete upstream regulator pRL120552, putR; see gene map in Fig. S1a) was amplified using the primers listed in supporting Table 1 with Phusion High-Fidelity DNA Polymerase (Thermo Fisher). Fragments were purified and double digested with $K p n I$ (at the 5' end) and BamHI (at the 3' end). Restriction fragments were cloned in front of the lux CDABE operon in the lux biosensor vector pIJ11268 (Frederix et al. 2014) to generate the proline biosensor plasmid pOPS0238 (Fig. S1b). As a positive control, the neomycin promoter producing constitutive luminescence was cloned upstream of lux genes into the same plasmid, generating pIJ11282. The promoterless lux vector pIJ11268 was used as a negative control. Plasmids were transferred into wild-type $R$. leguminosarum bv. viciae 3841 by triparental mating as previously described (Pini et al. 2017). All plasmids are available from Addgene (https://www.addgene.org).
Free-living assays

Bacterial strains were grown for 3 days on an UMA (30 mM sodium pyruvate and $10 \mathrm{mM}$ ammonium chloride) slope, resuspended in UMS without carbon or nitrogen and washed three times. Then cells were grown in UMS with $30 \mathrm{mM}$ sodium pyruvate and $10 \mathrm{mM}$ ammonium chloride with antibiotics for $16 \mathrm{~h}$. These cultures were then used as inocula for subsequent induction experiments during $3 \mathrm{~h}$. In these experiments, cells were grown in $10 \mathrm{~mL}$ UMS with different supplements (as specified in the corresponding figure). Luminescence (expressed as relative luminescence units, RLU) and $\mathrm{OD}_{600}$ were measured using the GloMaxMulti + Detection System (Promega). Specific luminescence was calculated as RLU per $\mathrm{OD}_{600}$.

Plant growth conditions

Pea (Pisum sativum var. Avola) seeds were surface sterilized and germinated on distilled water agar $(0.8 \%, \mathrm{w} / \mathrm{v})$ plates for 5 days in the dark at room temperature. Seedlings were then transferred to $13-\mathrm{cm}$ square Petri plates containing Fahräeus agar (Somasegaran et al. 1994) covered with sterile filter paper (one seedling per plate), as previously described (Pini et al. 2017). Seedlings were then inoculated with the corresponding bacterial strains by pouring liquid inoculum to the roots, adjusted to an $\mathrm{OD}_{600}$ equivalent to $2 \times 10^{7} \mathrm{CFU} /$ per root. Plates were closed with the lid and covered with aluminium foil to prevent exposure of roots to light. Plants were grown in a growth chamber under controlled environmental conditions (23 ${ }^{\circ} \mathrm{C}$ temperature, 16-h/8-h day/night cycle). 
Application and physiological characterisation of water deficit and salt stress

Seven days after inoculation, plants were transferred to fresh Fahräeus agar plates. For control plants, plates were replaced every three days to keep adequate moisture and nutrient levels. To generate water-deficit conditions, plants were maintained in the same plates for seven days so that water was progressively depleted. For the salt stress treatment, plants were transferred to Fahräeus plates containing $150 \mathrm{mM} \mathrm{NaCl}$ at $15 \mathrm{dpi}$ for $24 \mathrm{~h}$. In both cases, plants were transferred to fresh Fahräeus plates at 16 dpi and further grown for 5 days (recovery). Plates were analysed at 4,7 , $10,13,15$, and then daily until $21 \mathrm{dpi}$.

To establish the effect of water-deficit and salt stress on plants, stomatal conductance, leaf water potential ( $\left.\Psi_{\text {leaf }}\right)$ and net photosynthesis were measured $2 \mathrm{~h}$ after the beginning of photoperiod in the youngest fully expanded leaf. Stomatal conductance and net photosynthesis were measured with a portable open system mode (model LCpro+; ADC BioScientific Ltd.) using an ADC PLC-7504 leaf chamber. $\Psi_{\text {leaf }}$ was measured using a pressure chamber (Scholander et al. 1965). Symbiotic nitrogen fixation was measured as apparent nitrogenase activity (ANA) according to the method described by Witty and Minchin (1998). $\mathrm{H}_{2}$ evolution of intact plants was measured in an open flow-through system under $\mathrm{N}_{2} / \mathrm{O}_{2}(79 \% / 21 \%)$ using an electrochemical $\mathrm{H}_{2}$ sensor (Qubit Systems). The $\mathrm{H}_{2}$ sensor was calibrated with high purity gases using a gas mixer flowing at the same rate as the sampling system $\left(500 \mathrm{~mL} \mathrm{~min}^{-1}\right)$.

Image acquisition

Plates were photographed using a NightOWL camera (Berthold Technologies) as previously described (Pini et al. 2017). Briefly, CCD images (1,024 by 1,024 pixels) of light output were exposed for $120 \mathrm{~s}$ and analysed with the imaging software IndiGO (Berthold Technologies). Data are expressed as counts per second (cps) or as the

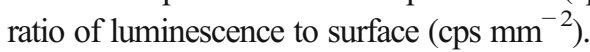

\section{Results}

The proline biosensor OPS0650 is able to detect concentrations of proline in the nanomolar range

The gene pRL120553 (hypothetical protein) and pRL120554 (putA) was shown to be induced 16-fold and 2-fold respectively in $R$. leguminosarum bv. viciae 3841 when grown in the presence of proline (Ramachandran et al. 2011). The gene putA encodes the putative multifunctional proline utilisation enzyme A (PutA), which combines ProDH and P5CDH activities, responsible for proline catabolisation in gramnegative bacteria (Liu et al. 2017). pRL120553 codes for a protein of unknown function and protein-BLAST of the predicted protein analysis do not show significant homology with previously described proteins. The gene $p u t R$, a putative AsnC family transcriptional regulator (pRL120552) is located upstream of pRL120553. These genes are located very close to one another, with intergenic regions around $50 \mathrm{nt}$ each (Fig. S1a). Thus, to generate a proline biosensor strain, a 605 bp region upstream of pRL120553 from $R$. leguminosarum bv. viciae 3841 was cloned into the lux vector pIJ11268 in front of the lux operon (Fig. S1b). The fragment cloned included the putative transcriptional regulator $p u t R$, since, from our experience, including the divergent regulator has been shown to improve the sensitivity of the promoter fusions (Pini et al. 2017). Transformation of $R$. leguminosarum bv. viciae 3841 with this plasmid generated the proline biosensor strain OPS0650 (Table 1).

To determine the specificity of the induction of lux expression in the biosensor, bacterial cells were grown in UMS supplemented with different compounds and luminescence was measured after $3 \mathrm{~h}$ of incubation (Fig. 1). Specific luminescence data are represented as relative luminescence units per optical density at $600 \mathrm{~nm}$ (RLU/OD 600 ) to account for the differences in bacterial growth. In all tests, strain LMB542 containing the empty vector pIJ11268 and the strain D5250 with constitutive lux expression were included as negative and positive controls, respectively. The proline biosensor showed luminescence values 86 -fold higher in the presence of $500 \mu \mathrm{M}$ of proline than those to cells grown in control medium. Cells grown in media supplemented with $10 \mathrm{mM}$ of L-4-hydroxyproline, a closely related non-proteinogenic amino acid, presented luminescence values comparable to those of a culture grown in the absence of proline, suggesting that the luminescence recorded is specific for proline. When pyruvate was eliminated from the media, the luminescence showed a decline, yet was still 17-fold higher than that of cells grown in the absence of the amino acid (Fig. 1). 
To define the limit of detection of the biosensor, the strain was incubated in UMS media supplemented with different proline concentrations ranging from $1 \mathrm{nM}$ to $500 \mu \mathrm{M}$ (Fig. 1). The biosensor was able to detect concentrations of proline as low as $300 \mathrm{nM}$, showing a significant specific luminescence of around $4.42 \times 10^{5} \pm$ $0.82 \times 10^{5} \mathrm{RLU} / \mathrm{OD}_{600}$. Increases in the concentration of proline over $50 \mu \mathrm{M}$ did not produce significant increments in luminescence emission, maintaining luminescence values of approximately $5.63 \times 10^{6} \pm 8.22 \times$ $10^{5} \mathrm{RLU} / \mathrm{OD}_{600}$.

In vivo monitorisation of proline in root exudates and nodules

We then analysed the spatial and temporal expression of the lux reporter in plants inoculated with the biosensor strain during root growth and nodule development. Pea plants were inoculated with either the biosensor strain OPS0650 or the negative and positive control strains LMB542 and D5250, respectively. Luminescence was measured at different time points from 4 to 20 dpi (Fig. 2). We noted that the hypocotyl showed background luminescence most likely due to the presence of chlorophyll in the upper part of the root in proximity to the light. Thus, to measure luminescence in roots we selected the area of the root corresponding to a length of $2-3 \mathrm{~cm}$ per root width, located aprox. $2 \mathrm{~cm}$ from the hypocotyl to prevent the above-mentioned background luminescence. To calculate luminescence in nodules we used the area corresponding to single nodules. No proline was added to the agar plates and the light output measured in plant roots inoculated with the negative control strain showed average values of 2.90 $\pm 0.18 \mathrm{cps} \mathrm{mm}^{-2}$ across the experiment (Fig. S2; Fig. S3). Therefore, the differences in luminescence detected can be attributed to either the proline present in root exudates or the proline detected by the differentiated nitrogen-fixing form of rhizobium, the bacteroids, within the infected nodule cells. Four days after inoculation, luminescence was induced mainly on primary roots, particularly in the upper half section of the main root (Fig. 2a). As lateral roots developed, luminescence could also be observed in the oldest lateral roots, although at relatively lower levels (Fig. 2b-f). These discrepancies may be related to the inoculation procedure, which was carried out when plants presented only a main root. At 10 dpi luminescence was detected in nodules (Fig. 2c), reaching maximum intensity values at 15 dpi (Fig. 2d).

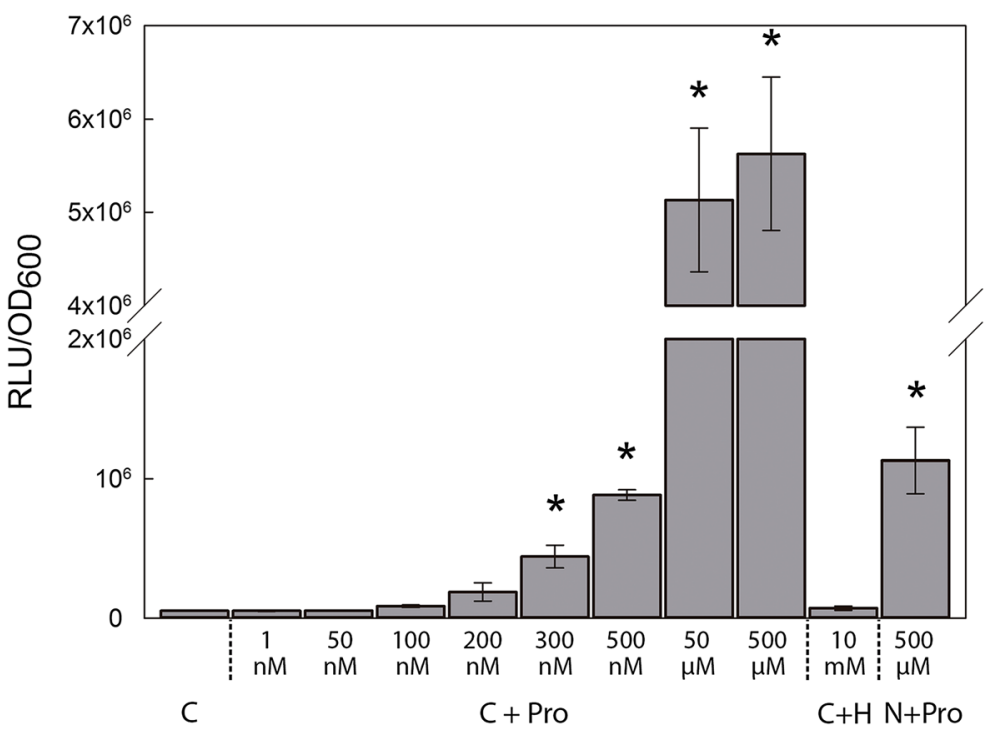

Fig. 1 Specificity and sensitivity of the proline biosensor strain OPS0650. Luminescence values are expressed as relative luminescence units (RLU) per optical density at $600 \mathrm{~nm}\left(\mathrm{OD}_{600}\right)$. Cells were grown using different combinations of universal minimal salts (UMS) media. $\mathrm{C}$ stands for control growth media containing $\mathrm{UMS}+30 \mathrm{mM}$ pyruvate $+10 \mathrm{mM}$ ammonium chloride $\left(\mathrm{NH}_{4} \mathrm{Cl}\right)$; $\mathrm{C}+$ Pro, control medium supplemented with various concentrations of proline as stated; $\mathrm{C}+\mathrm{H}$, control medium + $10 \mathrm{mM}$ L-4-hydroxyproline; $\mathrm{N}+$ Pro, UMS medium + $10 \mathrm{mM}$ $\mathrm{NH}_{4} \mathrm{Cl}+500 \mu \mathrm{M}$ Pro. Values represent mean $\pm \mathrm{SE}$ from two independent experiments in the case of $\mathrm{nM}$ proline concentrations and three independent experiments in the other measurements. An asterisk (*) indicates significant differences from C (Student's $t$ test at $\mathrm{p} \leq .05$ ) 
Induction of the proline biosensor occurs after a water-deficit and osmotic stress period

We then employed the luminescence-based proline biosensor strain to analyse the effect of gradual water loss and short-term salt stress on nodulated plants. Waterdeficit conditions were created by growing the plants on the same plates for seven days. Doing so, the water content of the agar medium, and therefore, the water available for the plant, was progressively depleted. To monitor the level of the water-deficit stress imposed, we checked the following physiological parameters: leaf water potential $\left(\Psi_{\text {leaf }}\right)$, stomatal conductance, net photosynthesis and symbiotic nitrogen fixation (Table 2). At this stage (16 dpi), $\Psi_{\text {leaf }}$ showed a significant decline, with values around $-0.49 \pm 0.1 \mathrm{MPa}$, while control plants maintained at optimal water conditions showed $\Psi_{\text {leaf }}$ values of $-0.24 \pm 0.05 \mathrm{MPa}$. Similarly, water deficit caused a $66 \%$ decline in stomatal conductance and a $36 \%$ reduction in photosynthetic rates compared to control plants. Regarding nitrogen fixation, water deficit and salt stress caused a 52 and $67 \%$ reduction in the rates of nitrogen fixation, respectively, compared to plants under optimal conditions (Table 2).

In regard to the effects of the different stress treatments on plants inoculated with the proline biosensor, initial experiments showed that the main changes in luminescence were observed in nodules, not in the root exudates. Therefore, analyses of the effect of the stresses are mostly focused on the luminescence of nodules alone. Nevertheless, we also analyzed the variations in luminescence in roots, selecting a region of the main root to facilitate comparisons across treatments. Plants inoculated with the proline biosensor OPS0650 showed significant changes in luminescence when compared with the negative control strain LMB542 (Fig. S3). However, as earlier observed, with the exception of samples at day 16 (24 h after the stress treatments), roots inoculated with biosensor strain did not show significant changes across the experiment.

Regarding the effect of the stresses on nodules, during the period of gradual water loss, the luminescence of nodules was maintained at relatively constant values, suggesting that proline levels did not show significant changes in bacteroids (Fig. 3a, Fig. S4). At 16 dpi, plants were

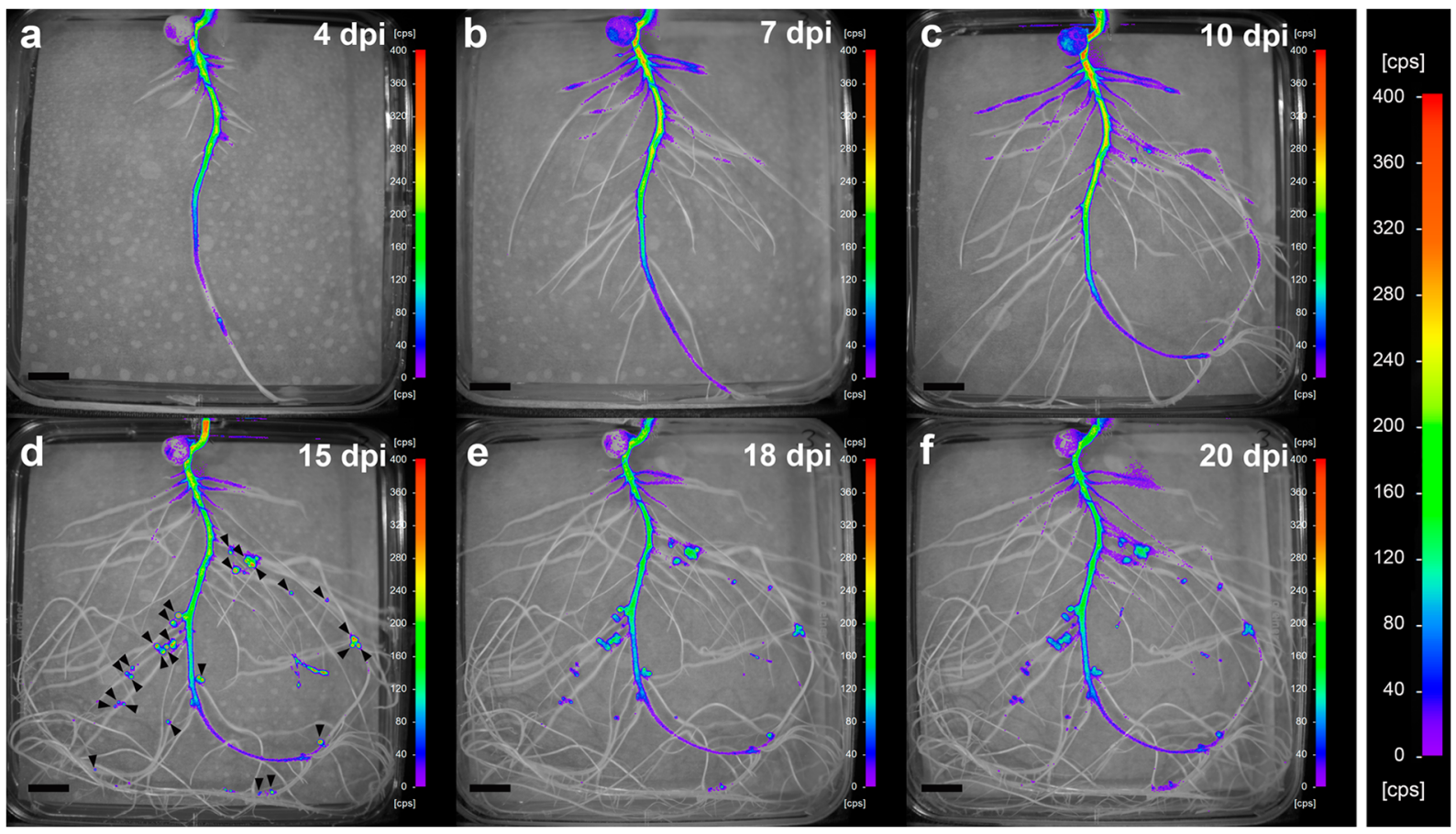

Fig. 2 In vivo temporal and spatial expression of the proline biosensor strain in pea roots and nodules. Images are representative of plates corresponding to 5 biological replicates and were acquired at 4 (a), 7 (b), 10 (c), 15 (d), 18 (e), and 20 (f) dpi.
Nodules were visible to the naked eye at around $10 \mathrm{dpi}$. Arrowheads were added in image (d) to show nodule position. Note that the same scale has been used in all images to facilitate comparisons. Scale bar, $1 \mathrm{~cm}$ 
Table 2 Effect of water deficit and osmotic stress on leaf water potential, stomatal conductance, photosynthesis and ANA of pea plants. Values represent the mean $\pm \mathrm{SE}(7 \leq \mathrm{n} \leq 10$ biological replicates, except in ANA with $n=4)$. An asterisk (*) indicates significant differences compared to control plants. (Student's $t$-test at $\mathrm{p} \leq .05)$. NDW, nodule dry weight

\begin{tabular}{lrrr}
\hline Parameter (Units) & \multicolumn{1}{c}{ Control } & Water deficit & Salinity \\
\hline Stomatal conductance $\left(\mathrm{mol} \mathrm{m} \mathrm{s}^{-2}\right.$ s & $0.09 \pm 0.01$ & $0.03 \pm 0.01^{*}$ & $0.04 \pm 0.01^{*}$ \\
Leaf water potential $(\mathrm{MPa})$ & $-0.24 \pm 0.05$ & $-0.49 \pm 0.1^{*}$ & $-0.39 \pm 0.03^{*}$ \\
Photosynthesis $\left(\mu \mathrm{mol} \mathrm{CO} \mathrm{m}^{-2} \mathrm{~s}^{-1}\right)$ & $7.07 \pm 0.38$ & $4.52 \pm 0.47^{*}$ & $4.73 \pm 0.26^{*}$ \\
ANA $\left(\mu \mathrm{mol} \mathrm{H} \mathrm{g} \mathrm{NDW}^{-1} \mathrm{~min}^{-1}\right)$ & $0.54 \pm 0.06$ & $0.26 \pm 0.01^{*}$ & $0.23 \pm 0.07^{*}$ \\
\hline
\end{tabular}

transferred to fresh plates (i.e., optimal water availability) as a recovery treatment. Recovery provoked a rapid increase in the luminescence observed in nodules within the first $24 \mathrm{~h}$, showing a gradual reduction in the following days (Fig. 3a and c, Fig. S4), almost reaching the levels of lux expression in nodules of control plants (Fig. 3a and b).

Salt stress was generated by incubating plants on plates containing $150 \mathrm{mM} \mathrm{NaCl}$ for one day. Similarly, to the water-deficit experiment, recovery was carried out by transferring the plants to fresh plates under optimal growth conditions, corresponding to the time point 16 dpi. Salinity also had a negative impact on the physiology of the plants, which showed $\Psi_{\text {leaf }}$ values of $-0.39 \pm$ $0.03 \mathrm{MPa}$, a $55 \%$ decline in stomatal conductance and a $35 \%$ reduction in net photosynthesis (Table 2). $24 \mathrm{~h}$ after the application of the salt stress there were no significant changes in the level of luminescence of the proline biosensor compared to the luminescence recorded before the onset of the treatment (Fig. 3a, Fig. S5). At $17 \mathrm{dpi}$, however, luminescence started to increase, reaching its maximum at 18 dpi (Fig. $3 \mathrm{a}$ and d, Fig. S5). Subsequently, the levels of luminescence progressively declined, yet were significantly higher than those of control nodules at the end of the experiment (Fig. 3a).

Nodules of plants inoculated with the negative control strain LMB542 showed negligible luminescence levels (Fig. 3e; Fig. S2), while nodules of plants inoculated with D5250, the strain carrying the constitutive lux expression plasmid, showed the maximum levels of luminescence (Fig. 3f; Fig. S6).

\section{Discussion}

This work describes a novel lux biosensor for the detection of proline. The biosensor takes advantage of the prolinespecific promoter of the gene pRL120553, a gene in close proximity to the gene pRL120554 (putA), of Rhizobium leguminosarum bv. viciae 3841, which were highly expressed during growth in proline. The induction assay showed that this biosensor is very sensitive as it can sense proline at concentrations as low as $300 \mathrm{nM}$. Also, testing with L-4-hydroxyproline, a close relative of proline, showed that this biosensor is very specific to proline. Currently, this biosensor is housed in Rhizobium leguminosarum bv. viciae 3841, although it should work in other related rhizobia. This novel construct allows the semi-quantitative estimation of the levels of proline both secreted by roots in the rhizosphere as well as the proline accessible for bacteroids inside nodules. The measurement is carried out in a non-invasive manner, maintaining tissue integrity, and thus, avoiding possible artefacts or degradation issues faced when collecting root exudates or isolating symbiosomes. Additionally, it allows the monitoring of proline levels across time using a simple plate growth assay. Although lux-based systems come with their own limitations (i.e., dependence on oxygen, ATP or reducing power; Brodl et al. 2018), this type of $l u x$-based biosensor has been successfully used for the in vivo monitoring of a number of metabolites including sugars, polyols and organic acids (Pini et al. 2017), as well as signalling compounds such as rhizopines (Geddes et al. 2019).

In terms of root secretion, proline was detected mainly on the primary root and, at later stages, with lateral roots and nodules (Fig. 2, Fig. S2, Fig. S3). This luminescence was shown to be specific of the presence of proline since plants inoculated with the negative control strain showed negligible light output (Fig. S2, Fig. S3). Interestingly, these regions do not correspond to root zones that are preferentially colonised by rhizobia (i.e., the elongation zone), neither co-localise with the secretion of other metabolites such as the amino acid phenylalanine or carbohydrates like sucrose (Pini et al. 2017). This suggests that the composition of the root exudates varies depending on the root zones, an observation that could not be made using classical root exudate extraction approaches. 

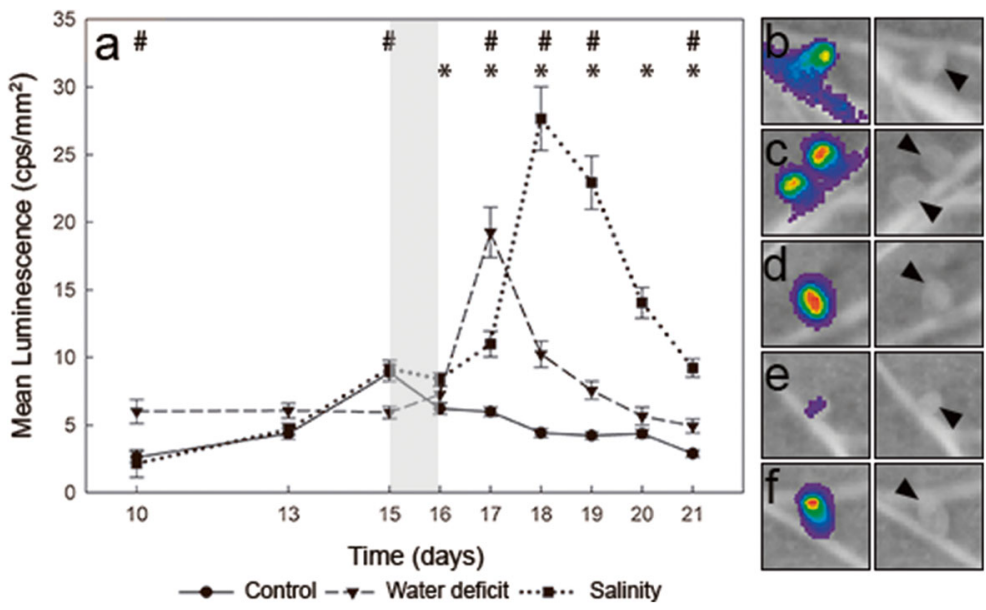

Fig. 3 a In vivo monitoring of proline in pea nodules of plants inoculated with the biosensor $R$. leguminosarum bv. viciae strain OPS650. Values represent mean luminescence [counts per second (cps) $\mathrm{mm}^{-2}$ ] from nodules of pea plants inoculated with the proline biosensor. Stress was induced at $15 \mathrm{dpi}$ for $24 \mathrm{~h}$ (grey area). For recovery, plants were transferred to fresh plates (optimal growth conditions). Values represent the mean $\pm \mathrm{SE}$ calculated from 5 biological replicates using the luminescence values of all the nodules present in a plant. An asterisk or a hash sign (* or \#) indicate significant differences between salt-stressed or waterdeficit plants and control plants, respectively (ANOVA at $\mathrm{p}$ $<.05$, Dunnett T3 post hoc test). (b) to (f) representative images from nodules of pea plants under different conditions, and their

Symbiotic root nodules represent a strong sink tissue for the plant, requiring the transport of high levels of sucrose to fuel nitrogenase activity and the biosynthesis of a large number of proteins and other biomolecules to maintain the high rates of metabolic activities. Once inside nodules, the major energy source provided by the plant to the bacteroids is in the form of malate (Udvardi et al. 1988; Ronson et al. 1981; Driscoll and Finan 1993). However, the peribacteroid membrane also allows the transport of amino acids, including proline (Udvardi et al. 1990; Zhu et al. 1992). This fact, along with the observation of increased proline degradation activity under drought and salt stress conditions (Kohl et al. 1988), has led to suggest that besides its osmoprotectant role, proline can also be used as a carbon, nitrogen and energy source for bacteroids (Curtis et al. 2004; Kohl et al. 1994). Indeed, exogenous application of proline or inoculation with a strain overexpressing putA has been shown to improve nitrogen fixation under drought stress conditions (Kohl et al. 1994; Zhu et al. 1992; van Dillewijn et al. 2001).

The current work shows that proline levels increased within bacteroids during nodule development, reaching a corresponding luminescence image. b nodules of plants inoculated with the proline biosensor strain OPS0650 under control conditions (17 dpi, scale $0-400 \mathrm{cps}$ ); c nodules from water-deficit plants at the maximum level of luminescence of the proline biosensor during recovery (17 dpi, scale $0-1100 \mathrm{cps}$ ); d nodules from saltstressed plants at the maximum luminescence of the proline biosensor during recovery (18 dpi, scale $0-3000 \mathrm{cps}$ ); e nodules from plants inoculated with the negative control strain LMB542 (no lux expression, $17 \mathrm{dpi}$, scale $0-400 \mathrm{cps}$ ); f nodules from plants inoculated with the positive control strain D5250 (constitutive lux expression,17 dpi, scale 1200-25200 cps). Arrowheads were added in images (b) to (f) to show nodule position

maximum level at $15 \mathrm{dpi}$. It is also interesting to note that, based on the luminescence detected, proline levels in nodules are significantly higher than those detected in root exudates. However, in contrast to the classical stressinduced accumulation of proline in nodules (Fougere et al. 1991; Gil-Quintana et al. 2013; Larrainzar et al. 2009), proline levels in pea bacteroids did not show significant variations during the stress period, but during the recovery phase. Although there are several factors that could account for these discrepancies such as the duration or the intensity of the stress treatment, it is noteworthy that this is the first time that proline levels are measured in bacteroids within intact nodules (i.e., without disrupting the tissue). Thus, the increase in proline previously reported may account, at least partially, to the proline accumulation in the plant fraction of nodules. In this scenario, recuperation of optimal growth conditions during recovery may lead to a reactivation of bacteroid proline catabolism and/or increased import of proline from the cytosol to the symbiosomes. This activation of proline catabolism upon recovery has, to our knowledge, been so far only described in plants, where the expression of proline dehydrogenase is suppressed during osmotic stress but induced again upon 
the relief of the stress (Mani et al. 2002; Satoh et al. 2002). Proline uptake has been shown to occur through a diffusive process in bacteroids from alfalfa and soybean nodules (Trinchant et al. 1998; Pedersen et al. 1996; Udvardi et al. 1990). Thus, one possibility is that at least a fraction of the proline accumulated during the stress in the plant fraction catabolised by the bacteroid with a two-fold benefit: to facilitate restoring pre-stress proline levels in the cytosol of infected cells, and to provide an additional source of energy, carbon or nitrogen for bacteroid metabolism. In this regard, in experiments with bacteroids isolated from Vicia faba nodules, salt stress produced an accumulation of proline in the peribacteroid space, suggesting that symbiosomes may behave as osmometers to accommodate the osmotic changes occurring in infected cells (Trinchant et al. 1998). Based on results presented here, this could also be the situation in nodules subjected to water deficit. It would be of great interest to combine multiple biosensors driving the expression of, for instance, different fluorescent proteins so that the levels of key metabolites can be simultaneously monitored in vivo.

Acknowledgements This work was supported by grants AGL2014-56561-P and RTI2018-09463-B-C22 (MCIU/AEI/ FEDER, UE) and the Biotechnology and Biological Sciences Research Council [grant number BB/K001868/2]. M.I.R. received a predoctoral F.P.I. fellowship (BES-2012-059972) and a mobility grant (EEBB-C-15-00615) from the Spanish Ministry of Economy and Competitiveness. E.L. is a Ramón y Cajal fellow funded by the Spanish Ministry of Science and Innovation (grant RYC2018023867-I).

Open Access This article is licensed under a Creative Commons Attribution 4.0 International License, which permits use, sharing, adaptation, distribution and reproduction in any medium or format, as long as you give appropriate credit to the original author(s) and the source, provide a link to the Creative Commons licence, and indicate if changes were made. The images or other third party material in this article are included in the article's Creative Commons licence, unless indicated otherwise in a credit line to the material. If material is not included in the article's Creative Commons licence and your intended use is not permitted by statutory regulation or exceeds the permitted use, you will need to obtain permission directly from the copyright holder. To view a copy of this licence, visit http://creativecommons.org/licenses/by/4.0/.

\section{References}

Aspinall D, Paleg L (1981) Proline accumulation: physiological aspects. In: Paleg L, Aspinall D (eds) Physiology and biochemistry of drought resistance in plants. Academic Press, New York, pp 205-240
Bais HP, Weir TL, Perry LG et al (2006) The role of root exudates in rhizosphere interactions with plants and other organisms. Annu Rev Plant Biol 57:233-266. https://doi.org/10.1146 /annurev.arplant.57.032905.105159

Beringer JE (1974) R factor transfer in Rhizobium leguminosarum. Microbiology 84:188-198. https://doi.org/10.1099 /00221287-84-1-188

Brodl E, Winkler A, Macheroux P (2018) Molecular mechanisms of bacterial bioluminescence. Comput Struct Biotechnol J 16: 551-564. https://doi.org/10.1016/j.csbj.2018.11.003

Curtis J, Shearer G, Kohl DH (2004) Bacteroid proline catabolism affects $\mathrm{N}_{2}$ fixation rate of drought-stressed soybeans. Plant Physiol 136:3313-3318. https://doi.org/10.1104 /pp.104.044024

Driscoll BT, Finan TM (1993) NAD $^{+}$-dependent malic enzyme of Rhizobium meliloti is required for symbiotic nitrogen fixation. Mol Microbiol 7:865-873. https://doi.org/10.1111 j.1365-2958.1993.tb01177.x

Fougere F, Le Rudulier D, Streeter JG (1991) Effects of salt stress on amino acid, organic acid, and carbohydrate composition of roots, bacteroids, and cytosol of alfalfa (Medicago sativa L.). Plant Physiol 96:1228-1236

Frederix M, Edwards A, Swiderska A et al (2014) Mutation of praR in Rhizobium leguminosarum enhances root biofilms, improving nodulation competitiveness by increased expression of attachment proteins. Mol Microbiol 93:464-478. https://doi.org/10.1111/mmi.12670

Geddes BA, Paramasivan P, Joffrin A et al (2019) Engineering transkingdom signalling in plants to control gene expression in rhizosphere bacteria. Nat Commun 10:3430. https://doi. org/10.1038/s41467-019-10882-x

Gil-Quintana E, Larrainzar E, Arrese-Igor C, González EM (2013) Is $\mathrm{N}$-feedback involved in the inhibition of nitrogen fixation in drought-stressed Medicago truncatula? J Exp Bot 64:281292. https://doi.org/10.1093/jxb/ers334

Hasegawa PM, Bressan RA, Zhu J-K, Bohnert HJ (2000) Plant cellular and molecular responses to high salinity. Annu Rev Plant Physiol Plant Mol Biol 51:463-499. https://doi. org/10.1146/annurev.arplant.51.1.463

Huang X-F, Chaparro JM, Reardon KF et al (2014) Rhizosphere interactions: root exudates, microbes, and microbial communities. Botany 92:267-275. https://doi.org/10.1139/cjb-20130225

Jiménez-Zurdo JI, van Dillewijn P, Soto MJ et al (1995) Characterization of a Rhizobium meliloti proline dehydrogenase mutant altered in nodulation efficiency and competitiveness on alfalfa roots. Mol Plant Microbe Interact 8:492-498

Jimenez-Zurdo JI, Garcia-Rodriguez FM, Toro N (1997) The Rhizobium meliloti putA gene: Its role in the establishment of the symbiotic interaction with alfalfa. Mol Microbiol 23: 85-93. https://doi.org/10.1046/j.1365-2958.1997.1861555.x

Keuntje B, Masepohl B, Klipp W (1995) Expression of the putA gene encoding proline dehydrogenase from Rhodobacter capsulatus is independent of NtrC regulation but requires an Lrp-like activator protein. J Bacteriol 177:6432-6439. https://doi.org/10.1128/JB.177.22.6432-6439.1995

Kohl DH, Schubert KR, Carter MB et al (1988) Proline metabolism in $\mathrm{N}_{2}$-fixing root nodules: energy transfer and regulation of purine synthesis. Proc Natl Acad Sci U S A 85:2036-2040

Kohl DH, Straub PF, Shearer G (1994) Does proline play a special role in bacteroid metabolism? Plant. Cell Environ 17:1257-1262 
Larrainzar E, Wienkoop S, Scherling C et al (2009) Carbon metabolism and bacteroid functioning are involved in the regulation of nitrogen fixation in Medicago truncatula under drought and recovery. Mol Plant Microbe Interact 22:15651576. https://doi.org/10.1094/MPMI-22-12-1565

Lee B, Jin YL, Avice J et al (2009) Increased proline loading to phloem and its effects on nitrogen uptake and assimilation in water-stressed white clover (Trifolium repens). New Phytol 182: 654-663. https://doi.org/10.1111/j.1469-8137.2009.02795.x

Liu L-K, Becker DF, Tanner JJ (2017) Structure, function, and mechanism of proline utilization A (PutA). Arch Biochem Biophys 632:142-157. https://doi.org/10.1016/J. ABB.2017.07.005

Mani S, Van De Cotte B, Van Montagu M, Verbruggen N (2002) Altered levels of proline dehydrogenase cause hypersensitivity to proline and its analogs in Arabidopsis. Plant Physiol 128:73-83. https://doi.org/10.1104/PP.010572

Oburger E, Jones DL (2018) Sampling root exudates - Mission impossible? Rhizosphere 6:116-133. https://doi. org/10.1016/j.rhisph.2018.06.004

Olanrewaju OS, Ayangbenro AS, Glick BR, Babalola OO (2019) Plant health: feedback effect of root exudates-rhizobiome interactions. Appl Microbiol Biotechnol 103:1155-1166. https://doi.org/10.1007/s00253-018-9556-6

Oldroyd GED, Murray JD, Poole PS, Downie JA (2011) The rules of engagement in the legume-rhizobial symbiosis. Annu Rev Genet 45:119-144. https://doi.org/10.1146/annurev-genet110410-132549

Pedersen AL, Feldner HC, Rosendahl L (1996) Effect of proline on nitrogenase activity in symbiosomes from root nodules of soybean (Glycine max L.) subjected to drought stress. J Exp Bot 47:1533-1539. https://doi.org/10.1093/jxb/47.10.1533

Pini F, East AK, Appia-Ayme C et al (2017) Bacterial biosensors for in vivo spatiotemporal mapping of root secretion. Plant Physiol 174:1289-1306. https://doi.org/10.1104/pp.16.01302

Poole PS, Schofiel NA, Reid CJ et al (1994) Identification of chromosomal genes located downstream of $d c t D$ that affect the requirement for calcium and the lipopolysaccharide layer of Rhizobium leguminosarum. Microbiology 140:27972809. https://doi.org/10.1099/00221287-140-10-2797

Ramachandran VK, East AK, Karunakaran R et al (2011) Adaptation of Rhizobium leguminosarum to pea, alfalfa and sugar beet rhizospheres investigated by comparative transcriptomics. GenomeBiol 12:R106. https://doi.org/10.1186/gb-2011-12-10-r106

Rilling JI, Acuña JJ, Nannipieri P et al (2019) Current opinion and perspectives on the methods for tracking and monitoring plant growth-promoting bacteria. Soil Biol Biochem 130: 205-219. https://doi.org/10.1016/J.SOILBIO.2018.12.012

Ronson CW, Lyttleton P, Robertson JG (1981) C4-dicarboxylate transport mutants of Rhizobium trifolii form ineffective nodules on Trifolium repens. Proc Natl Acad Sci 78:4284-4288. https://doi.org/10.1073/pnas.78.7.4284

Satoh R, Nakashima K, Seki M et al (2002) ACTCAT, a novel cisacting element for proline- and hypoosmolarity-responsive expression of the proDH gene encoding proline dehydrogenase in Arabidopsis. Plant Physiol 130:709-719. https://doi. org/10.1104/PP.009993

Scholander PF, Bradstreet ED, Hemmingsen EA et al (1965) Sap pressure in vascular plants. Science 148:339-346. https://doi. org/10.1126/SCIENCE.148.3668.339
Somasegaran P, Hoben HJ, Heinz J (1994) Handbook for rhizobia: methods in legume-Rhizobium technology. Springer, New York

Szabados L, Savouré A (2010) Proline: a multifunctional amino acid. Trends Plant Sci 15:89-97

Tanner JJ (2008) Structural biology of proline catabolism. Amino Acids 35:719-730. https://doi.org/10.1007/s00726-008-0062-5

Trinchant JC, Yang YS, Rigaud J (1998) Proline accumulation inside symbiosomes of faba bean nodules under salt stress. Physiol Plant 104:38-49. https://doi.org/10.1034/j.13993054.1998.1040106.x

Turner TR, James EK, Poole PS (2013) The plant microbiome. Genome Biol 14:209. https://doi.org/10.1186/gb-2013-14-6-209

Udvardi M, Poole PS (2013) Transport and metabolism in legumerhizobia symbioses. Annu Rev Plant Biol 64:781-805. https://doi.org/10.1146/annurev-arplant-050312-120235

Udvardi MK, Price GD, Gresshoff PM, Day DA (1988) A dicarboxylate transporter on the peribacteroid membrane of soybean nodules. FEBS Lett 231:36-40. https://doi. org/10.1016/0014-5793(88)80697-5

Udvardi MK, Ou Yang L-J, Young S, Day DA (1990) Sugar and amino acid transport across symbiotic membranes from soybean nodules. Mol Plant-Microbe Interact 3:334-340. https://doi.org/10.1094/MPMI-3-334

van Dillewijn P, Soto MJ, Villadas PJ, Toro N (2001) Construction and environmental release of a Sinorhizobium meliloti strain genetically modified to be more competitive for alfalfa nodulation. Appl Environ Microbiol 67:3860 3865. https://doi.org/10.1128/aem.67.9.3860-3865.2001

van Overbeek LS, van Elsas JD (1995) Root exudate-induced promoter activity in Pseudomonas fluorescens mutants in the wheat rhizosphere. Appl Environ Microbiol 61:890-898

Verdoy D, Coba de la Peña T, Redondo FJ et al (2006) Transgenic Medicago truncatula plants that accumulate proline display nitrogen-fixing activity with enhanced tolerance to osmotic stress. Plant Cell Environ 29:1913-1923. https://doi. org/10.1111/j.1365-3040.2006.01567.x

Vives-Peris V, Molina L, Segura A et al (2018) Root exudates from citrus plants subjected to abiotic stress conditions have a positive effect on rhizobacteria. J Plant Physiol 228:208-217. https://doi.org/10.1016/J.JPLPH.2018.06.003

Webb BA, Hildreth S, Helm RF, Scharf BE (2014) Sinorhizobium meliloti chemoreceptor McpU mediates chemotaxis toward host plant exudates through direct proline sensing. Appl Environ Microbiol 80:3404-3415. https://doi.org/10.1128/AEM.00115-14

Witty JF, Minchin FR (1998) Methods for the continuous measurement of $\mathrm{O}_{2}$ consumption and $\mathrm{H}_{2}$ production by nodulated legume root systems. J Exp Bot 49:1041-1047. https://doi. org/10.1093/jxb/49.323.1041

Zhu Y, Shearer G, Kohl DH (1992) Proline fed to intact soybean plants influences acetylene reducing activity and content and metabolism of proline in bacteroids. Plant Physiol 98:1020 1028. https://doi.org/10.1104/PP.98.3.102

Publisher's Note Springer Nature remains neutral with regard to jurisdictional claims in published maps and institutional affiliations. 